\title{
Identification of an Arabidopsis thaliana Mutation (vsm1) That Restricts Systemic Movement of Tobamoviruses
}

\author{
Robert T. Lartey, Soumitra Ghoshroy, and Vitaly Citovsky \\ Department of Biochemistry and Cell Biology, State University of New York, Stony Brook 11794-5215, \\ U.S.A. \\ Accepted 26 March 1998.
}

\begin{abstract}
Following inoculation, many plant viruses spread locally from cell to cell until they reach the vascular system, through which they then move to other parts of the plant, resulting in systemic infection. To isolate host genes involved in systemic transport of plant viruses, ethyl methanesulfonate-mutagenized Arabidopsis thaliana plants were screened for significant delays in the systemic movement of turnip vein clearing virus (TCVC). One such mutant, designated vsm1 (virus systemic movement), was identified. Unlike the wild-type plants, vsm1 did not develop viral disease and did not allow the systemic spread of the virus. The local viral movement within the inoculated vsml leaves, however, was not affected. TVCV systemic movement within the vsm1 plants was likely blocked at the step of viral entry into the host plant vasculature from the infected leaf tissue. vsm1 plants also restricted the systemic movement of another tobamovirus but not of an unrelated carmovirus.
\end{abstract}

Our present understanding of the mechanisms by which viruses spread within their plant hosts is derived from studies of viral proteins involved in this process, i.e., coat and movement proteins. Little is known about plant factors required for viral movement. One approach to identify and characterize these putative host components is to isolate plant mutants defective for viral spread. Until now, no such mutants in Arabidopsis thaliana have been described although several ecotypes of this plant were found resistant to infection by cauliflower mosaic virus (CaMV) (Leisner et al. 1993), geminiviruses (Lee et al. 1994), and turnip crinkle virus (TCV; Simon et al. 1992; Dempsey et al. 1997). Furthermore, one A. thaliana mutation, toml, suggested to inhibit tobamoviral spread (Ishikawa et al. 1991) was later shown to interfere with viral replication instead (Ishikawa et al. 1993). Here, we report the first example of an A. thaliana mutant with a specific block in systemic movement of turnip vein clearing virus (TVCV).

TVCV is a positive-sense RNA tobamovirus that is

Corresponding author: Vitaly Citovsky; Telephone: 1-516-632-9534; Fax: 1-516-632-8575; E-mail: vitaly.citovsky@ sunysb.edu serologically related to tobacco mosaic virus (TMV) (Lartey et al. 1993). Recent experiments showed that TVCV efficiently infects A. thaliana, resulting in formation of clear disease symptoms but allowing survival and fertility of the infected plants (Lartey et al. 1997). Here, TVCV infection of A. thaliana was used as a model system to study virus-plant interaction. Specifically, we searched for A. thaliana mutants that do not support the systemic spread of TVCV and, thus, lack the symptoms of the viral disease. Twenty-five thousand plants derived from ethyl methanesulfonate (EMS)-mutagenized $\mathrm{M}_{2}$ seeds of Columbia ecotype of $A$. thaliana (Lehle Seeds, Round Rock, TX) were grown and mechanically inoculated with TVCV at the four-leaf stage as described previously (Lartey et al. 1997). The growth patterns of the infected mutant plants were compared with those of the inoculated wild-type A. thaliana. Asymptomatic plants were reinfected to compensate for occasional unsuccessful inoculations and scored again. To date, we have isolated one mutant that did not display the symptoms of viral infection. Figure 1 shows that TVCV infection retarded the growth of the wild-type plants (compare plants [u] and [i] in Figure 1A). In contrast, the growth of the identified $A$. thaliana mutant was unaffected by inoculation with TVCV (Fig. 1B), suggesting that this mutation altered the process of TVCVhost interaction.

We tested whether the mutant plants were able to support local (cell-to-cell) and/or systemic movement of TVCV. Local movement within the inoculated leaf and systemic movement to other leaves were assessed with in situ hybridization to directly detect the viral genomic RNA in the inoculated and uninoculated tissues (Lartey et al. 1997). The results in Figure 2 demonstrate that TVCV spread within the inoculated leaf (Fig. 2B and C) and moved systemically from this leaf into the uninoculated upper leaves of a wild-type A. thaliana plant (Fig. 2D). In the mutant plants, however, TVCV accumulated only in the inoculated leaf (Fig. 3F and G), leaving the uninoculated leaves free of the virus (Fig. $3 \mathrm{H}$ ). No difference in viral spread within the inoculated leaf was detected between the wild-type and the mutant plants (Fig. 2B and $\mathrm{C}$ versus $F$ and $G$, respectively). These observations suggest that, while TVCV replicated and spread within the inoculated leaf, it could not move systemically. Thus, this A. thaliana 
mutation was designated vsml (virus systemic movement). Since viral systemic, rather than local, spread is thought to underlie the viral disease, the arrested systemic movement of the virus is consistent with the absence of the disease symptoms in TVCV-infected vsml plants (Fig. 1).

One of the hallmarks of TVCV infection is the accumulation of large amounts of the $17.5-\mathrm{kDa}$ viral coat protein (CP) within the infected host cells (Lartey et al. 1997). We utilized CP production as a simple assay for viral spread within the inoculated wild-type and vsml plants (Lartey et al. 1997). Quantification of the CP content in the uninoculated upper (cauline) leaves showed that, in the wild-type plants, the maximal amounts of $\mathrm{CP}$ accumulated 6 to 8 days post infection (Fig. 3A). No CP was detected in the uninoculated leaves of the $v s m 1$ plants for up to 40 days post infection (Fig. 3B). Thus, the vsml mutation likely blocked, rather than slowed, the process of TVCV systemic infection.
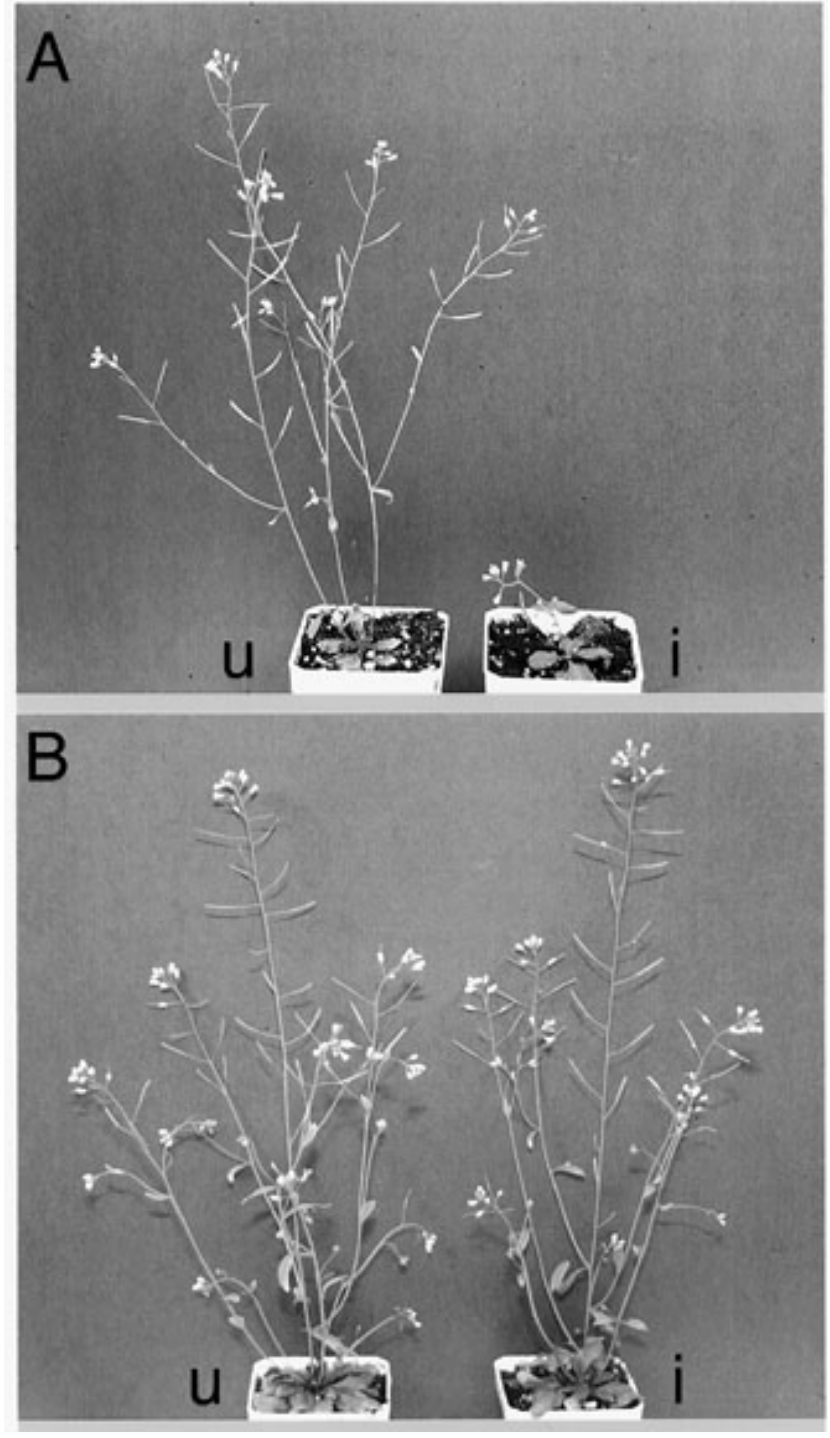

Fig. 1. Symptoms of turnip vein clearing virus (TVCV) infection in the wild-type and vsm1 Arabidopsis thaliana. Disease symptoms were observed 20 days after inoculation. A, Uninfected (u) and TVCVinfected (i) wild-type plants. B, Uninfected (u) and TVCV-infected (i) vsm1 plants.
Potentially, the vsml mutation could directly interfere with formation of infectious TVCV virions. In this case, the defective virus particles will be unable to exit the inoculated tissue and move systemically (Dawson et al. 1988; Saito et al. 1990; Hilf and Dawson 1993). To examine this possibility, the infectivity of TVCV virions assembled within the inoculated leaves of vsml A. thaliana plants was compared with that of TVCV particles derived from the wild-type tissue. Viral infectivity was quantified by the number and size of necrotic lesions induced in Nicotiana tabacum cv. Xanthi-nc, the local lesion host of TVCV (Lartey et al. 1993). Table 1 shows that no significant differences in either of these parameters were found between the wild-type and vsml plants. Also, the uninoculated leaves of TVCV-infected vsml contained no infectious viral particles (Table 1), confirming the lack of TVCV systemic movement in these plants. Importantly, all virus extracts used as inocula had been treated with RNase to remove any potential contamination with unencapsidated or partially-encapsidated TVCV RNA. Thus, our results demonstrate that TVCV virions that replicated and assembled within the inoculated leaves of vsml plants retain the full infectivity of the virus produced in the wild-type A. thaliana tissue.

The lack of TVCV systemic movement in vsml plants might be due to the inability of the virions either to enter the plant vasculature from the inoculated tissue or to exit the vascular system into uninoculated tissues. To distinguish between these two possibilities, we examined the presence of infectious TVCV virions in the stem internodes of the inoculated vsml plants. Because only minute quantities of the virus might be present in the vascular tissue, they might not be
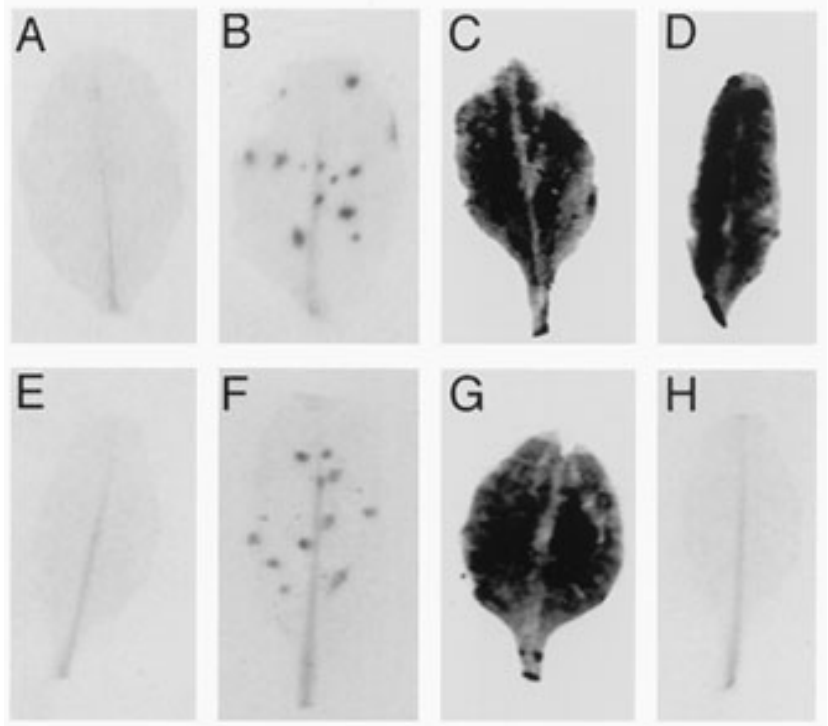

Fig. 2. Accumulation of turnip vein clearing virus (TVCV) RNA in inoculated but not in uninoculated leaves of the $v s m 1$ plant. (A-D) Wild-type and (E-H) vsm 1 plants were inoculated with TVCV. Two or 10 days after infection (d.p.i.), the inoculated and uninoculated leaves were harvested, and the presence of viral genomic RNA was determined by in situ hybridization with a ${ }^{33} \mathrm{P}$-labeled probe derived from TVCV cDNA (Lartey et al. 1994), followed by autoradiography (Lartey et al. 1997). A, E, Uninfected leaves. B, F, Inoculated leaves (2 d.p.i.). C, G, Inoculated leaves (10 d.p.i.). D, H, Uninoculated upper leaves (10 d.p.i.). 
detectable by the $\mathrm{CP}$ assay. However, even very small amounts of TVCV are sufficient to infect healthy host plants. Thus, we used the stem internodes, composed mostly of vascular tissue, as a source of inoculum to infect the local lesion host tobacco plants. Specifically, the internode samples located $1.0 \mathrm{~cm}$ away from the inoculated leaf were harvested 10 days after TVCV was inoculated on the wild-type or vsm 1 plants. The collected tissue was ground and used to infect $N$. tabacum cv. Xanthi-nc plants. Table 1 shows that while the plants inoculated with stem extracts from TVCV-infected, wild-type $A$. thaliana developed local lesions, no such lesions were detected on tobacco plants inoculated with vsml stem extracts, indicating that stem internodes from the vsml plants did not contain active virions. Thus, the $v s m 1$ mutation likely blocked viral entry from the inoculated tissue into the vasculature. Furthermore, the molecular mechanism of this initiation of systemic movement must be distinct from that of the local spread of the virus, which was not affected in the vsml plants (Fig. 2).

Plant viral infection is often characterized by a high specificity of virus-host interaction (reviewed by Simon 1994) Thus, if the vsml mutation interfered with a specific step of TVCV-host interaction, it might not affect the systemic spread of an unrelated virus. To test this idea, we inoculated the vsm I plants with TCV (kindly provided by S. Howell, Cornell University) and followed the time course of its systemic movement by the $\mathrm{CP}$ assay. Figure 3 shows that the vsm 1 mutation had no effect on accumulation of TCV CP and, by implication, TCV virions in the inoculated leaves. However, when the $v s m l$ plants were inoculated with the tomato strain of TMV, its systemic spread was arrested (Fig. 3). Because both TVCV and TMV belong to the phylogenic group of

Fig. 3. Effect of the vsm 1 mutation on the time course of turnip vein clearing virus (TVCV), turnip crinkle virus (TCV), and tobacco mosaic virus (TMV) coat protein (CP) accumulation in uninoculated leaves of the (A) wild-type and (B) vsml plants. Plants were infected and their leaves harvested at indicated time periods after infection and analyzed by sodium dodecyl sulfate-gel electrophoresis. Amounts of TVCV CP (open squares), TCV CP (filled circles), and TMV CP (open circles) were estimated by scanning densitometry of Coomassie blue-stained gels. Graphs represent an average of three independent experiments.

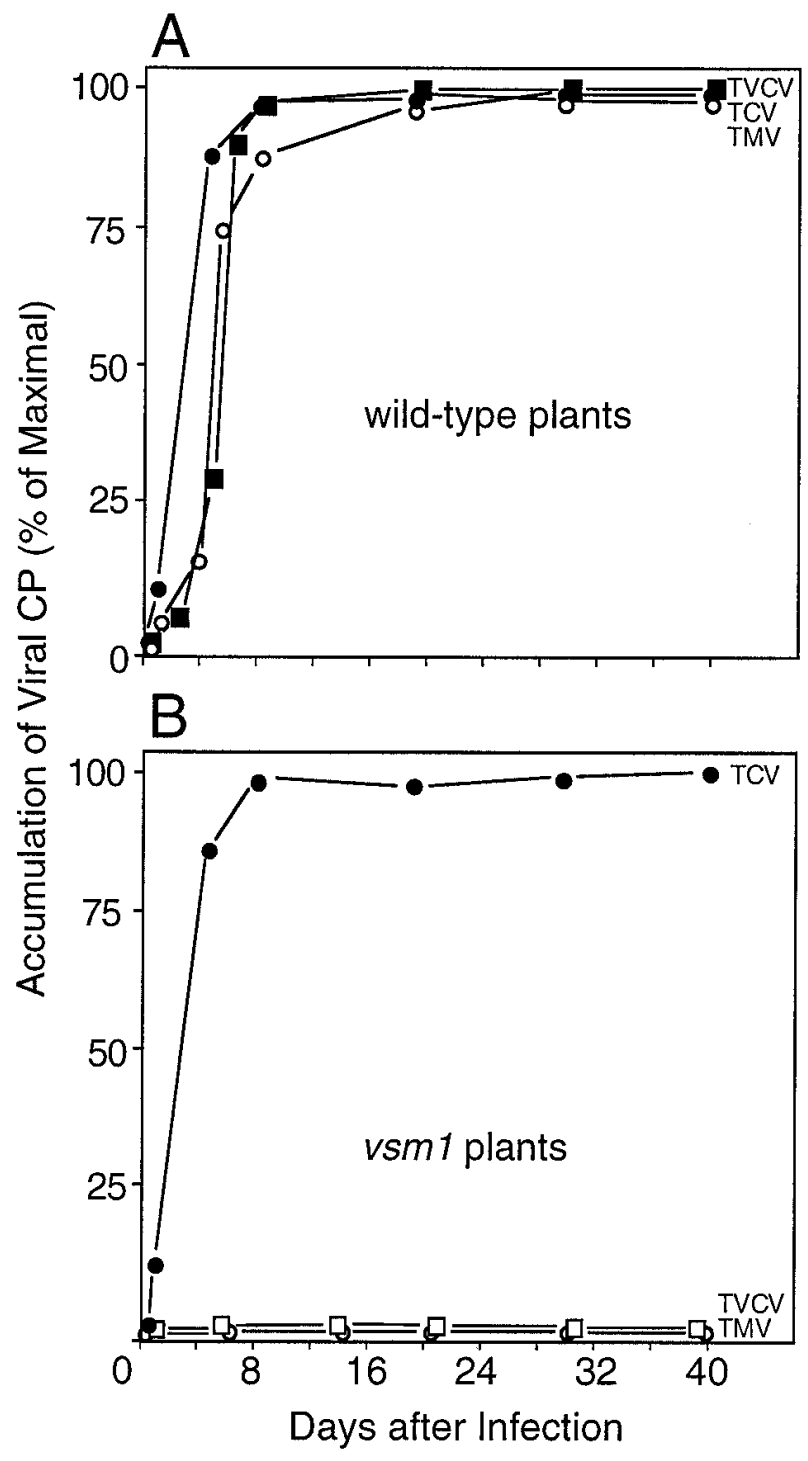

Table 1. Formation of infectious turnip vein clearing virus (TVCV) virions within the inoculated leaves of the wild-type and vsm1 Arabidopsis thaliana plants $^{\mathrm{a}}$

\begin{tabular}{lcccc}
\hline & & & \multicolumn{2}{c}{ Lesions } \\
\cline { 5 - 5 } Source of inoculum & Dilution of inoculum & Number $\pm \mathbf{S E}$ & Diameter $\pm \mathbf{S E}(\mathbf{m m})$ & Percentage of wild type (no./diameter) \\
\hline Wild-type inoculated leaf & $1: 100$ & $130 \pm 20$ & - & - \\
& $1: 1,000$ & $10 \pm 4$ & $3.6 \pm 1.4$ & $100 / 100$ \\
vsml inoculated leaf & $1: 100$ & $128 \pm 35$ & - & - \\
Wild-type uninoculated leaf & $1: 1,000$ & $11 \pm 5$ & $3.8 \pm 1.2$ & $98 / 105$ \\
& $1: 100$ & $131 \pm 22$ & - & - \\
vsml uninoculated leaf & $1: 1,000$ & $12 \pm 5$ & $3.5 \pm 1.2$ & - \\
Wild-type stem & $1: 100$ & 0 & - & - \\
& $1: 100$ & $124 \pm 180$ & $3.3 \pm 1.2$ & $100 / 100$ \\
vsml stem & $1: 1,000$ & $11 \pm 4$ & 0 & $0 / 100$ \\
\hline
\end{tabular}

a Ten days after inoculation, two 5-mm leaf disks or stem samples were collected from TVCV-infected wild-type and $v s m 1$ plants. Virions were extracted and used to inoculate Nicotiana tabacum cv. Xanthi-nc plants as described (Ghoshroy et al., in press). Number and size of local lesions are average of three infected tobacco plants in which lesions on four inoculated leaves per plant were counted and measured 10 days after inoc ulation; SE, standard error. 
tobamoviruses whereas TCV is an unrelated carmovirus, the vsml mutation may affect very specific steps in the tobamovirus-plant host interaction.

Genetic segregation analysis showed that the progeny of the homozygous vsm 1 plants crossed to the wild-type A. thaliana $\left(F_{1}\right)$ did not develop the mutant phenotype (i.e., resistance to TVCV systemic movement) following TVCV infection, indicating that $v s m l$ is a recessive mutation. When this $F_{1}$ population was self-pollinated, the resulting $F_{2}$ progeny segregated 3.1:1 $\left(\chi^{2}=0.12\right)$, suggesting vsm1 likely represents a mutation in a single genetic locus. However, the relatively small number of progeny tested does not rule out the possibility of two loci less than 12 map units apart.

How does the vsm1 mutation block viral movement? In plants, disease resistance is often expressed as a hypersensitive response (HR) that restricts pathogen spread following localized cell death (known as local lesions) at the sites of inoculation. The absence of such local lesions on the inoculated leaves of vsml plants suggested that the bona fide $\mathrm{HR}$ is not involved in the inhibition of TVCV systemic infection. In addition to the HR pathway, the rate of plant development has been shown to underlie resistance of several A. thaliana ecotypes to viral systemic infection. Specifically, faster developing plants became resistant to $\mathrm{CaMV}$ while slower development increased sensitivity to the virus (Leisner et al. 1993). However, we could not detect any differences in the rate of plant development between the vsml mutant and the wild-type plants, arguing against the developmental basis for the $v s m 1$ phenotype.

The bundle sheath-phloem parenchyma boundary has been suggested to represent the barrier that separates the local and systemic movement of plant viruses (Ding et al. 1992; Lucas and Gilbertson 1994; Thompson and García-Arenal 1998). It is possible, therefore, that the vsml mutant is defective in a protein function essential for the interaction of TVCV with plasmodesmata on this boundary between the vascular and nonvascular tissues. Because no viral material was detected in stems of the inoculated vsml plants, the systemic movement of TVCV was likely blocked at the stage of entering the vascular tissue and not egressing from it. Interestingly, TVCV systemic movement in tobacco plants was arrested by nontoxic concentrations of the heavy metal cadmium (Ghoshroy et al., in press). In that case, however, TVCV transport into the plant vasculature was not affected. Instead, cadmium treatment of the host plant blocked viral exit from the vasculature into uninoculated, nonvascular tissues (Ghoshroy et al. in press). Thus, viral systemic movement may be a polar process in which TVCV virions enter and exit the vascular system by two different mechanisms.

Interference with the plant factors required for viral systemic movement may involve essential cellular functions. This would explain why only one $v s m$ mutant was found in 25,000 EMS mutants that were screened or why such A. thaliana mutants have not been described previously. That vsml affected the spread of tobamoviruses, but not of an unrelated carmovirus, suggests multiple pathways for viral systemic movement. Most likely, only one such pathway is compromised in the vsml plants, allowing the survival of this mutant. In summary, our observations suggest that the vsml mutant may represent a new type of $A$. thaliana genes required for the systemic movement of tobamoviruses, potentially affecting the viral ability to cross transport boundaries between the nonvascular and vascular tissues.

\section{ACKNOWLEDGMENTS}

This work was supported by grants from National Institutes of Health (grant no. R01-GM50224), U.S. Department of Agriculture (grant no. 94-02564), and U.S.-Israel Binational Research and Development Fund (BARD) (grant no. US-2247-93) to V. C. R. T. L. is a recipient of a National Institutes of Health Grant Supplement award (grant no. GM46134).

\section{LITERATURE CITED}

Dawson, W. O., Bubrick, P., and Grantham, G. L. 1988. Modifications of the tobacco mosaic virus coat protein gene affecting replication, movement and symptomatology. Phytopathology 78:783-789.

Dempsey, D. A., Pathirana, M. S., Wobbe, K. K., and Klessig, D. F. 1997. Identification of an Arabidopsis locus required for resistance to turnip crinkle virus. Plant J. 11:301-311.

Ding, B., Haudenshield, J. S., Hull, R. J., Wolf, S., Beachy, R. N., and Lucas, W. J. 1992. Secondary plasmodesmata are specific sites of localization of the tobacco mosaic virus movement protein in transgenic tobacco plants. Plant Cell 4:915-928.

Ghoshroy, S., Freedman, K., Lartey, R., and Citovsky, V. Inhibition of plant viral systemic infection by non-toxic concentrations of cadmium. Plant J. (In press.)

Hilf, M. E., and Dawson, W. O. 1993. The tobamovirus capsid protein functions as a host-specific determinant of long-distance movement. Virology 193:106-114.

Ishikawa, M., Naito, S., and Ohno, T. 1993. Effects of the tom1 mutation of Arabidopsis thaliana on the multiplication of tobacco mosaic virus RNA in protoplasts. J. Virol. 67:5328-5338.

Ishikawa, M., Obata, F., Kumaga, T. I., and Ohno, T. 1991. Isolation of mutants of Arabidopsis thaliana in which accumulation of tobacco mosaic virus coat protein is reduced to low levels. Mol. Gen. Genet. 230:33-38.

Lartey, R., Ghoshroy, S., Ho, J., and Citovsky, V. 1997. Movement and subcellular localization of a tobamovirus in Arabidopsis. Plant J. 12: 537-545.

Lartey, R. T., Hartson, S. D., Pennington, R. E., Sherwood, J. L., and Melcher, U. 1993. Occurrence of a vein-clearing tobamovirus in turnip. Plant Dis. 77:21-24.

Lartey, R. T., Lane, L. C., and Melcher, U. 1994. Electron microscopic and molecular characterization of turnip vein-clearing virus. Arch. Virol. 138:287-298.

Lee, S., Stenger, D. C., Bisaro, D. M., and Davis, K. R. 1994. Identification of loci in Arabidopsis that confer resistance to geminivirus infection. Plant J. 6:525-535.

Leisner, S. M., Turgeon, R., and Howell, S. H. 1993. Effects of host plant development and genetic determinants on the long-distance movement of cauliflower mosaic virus in Arabidopsis. Plant Cell 5: 191-202.

Lucas, W. J., and Gilbertson, R. L. 1994. Plasmodesmata in relation to viral movement within leaf tissues. Annu. Rev. Phytopathol. 32:387411.

Saito, T., Yamanaka, K., and Okada, Y. 1990. Long distance movement and viral assembly of tobacco mosaic virus mutants. Virology 176: 329-336.

Simon, A. E. 1994. Interactions between Arabidopsis thaliana and viruses. Pages 685-704 in: Arabidopsis. Monograph 27. E. M. Meyerowitz and C. R. Somerville, eds. Cold Spring Harbor Laboratory, Cold Spring Harbor, NY.

Simon, A. E., Li, X. H., Lew, J. E., Stange, R., Zhang, C., Polacco, M., and Carpenter, C. D. 1992. Susceptibility and resistance of Arabidopsis thaliana to turnip crinkle virus. Mol. Plant-Microbe Interact. 5:496-503.

Thompson, J. R., and García-Arenal, F. G. 1998. The bundle sheathphloem interface of Cucumis sativus is a boundary to systemic infection by tomato aspermy virus. Mol. Plant-Microbe Interact. 11: 109-114. 